\title{
The discrediting of the monoamine hypothesis
}

Marty Hinz'

Alvin Stein ${ }^{2}$

Thomas Uncini ${ }^{3}$

'Clinical Research, NeuroResearch Clinics, Inc, Cape Coral, FL, ${ }^{2}$ Stein Orthopedic Associates, Plantation, FL, ${ }^{3}$ Fairview University Medical Center, Hibbing, MN, USA
Correspondence: Marty Hinz 1008 Dolphin Drive, Cape Coral, FL 33904, USA

$\mathrm{Tel}+\mathrm{I} 2186262220$

Fax +I 2186261638

Email marty@hinzmd.com
This article was published in the following Dove Press journal:

International Journal of General Medicine

13 February 2012

Number of times this article has been viewed

Background: The monoamine hypothesis has been recognized for over half a century as a reference point to understanding electrical dysfunction associated with disease states, and/or regulatory dysfunction related to synaptic, centrally acting monoamine concentrations (serotonin, dopamine, norepinephrine, and epinephrine).

Methods: Organic cation transporters (OCT) are a primary force controlling intracellular and extracellular (including synaptic) concentrations of centrally acting monoamines and their amino acid precursors. A new type of research was analyzed in this paper (previously published by the authors) relating to determining the functional status of the nutritionally driven organic cation transporters. It was correlated with the claims of the monoamine hypothesis.

Results: Results of laboratory assays from subjects not suffering from a hyperexcreting tumor show that centrally acting monoamine concentrations are indistinguishable in subjects with and without disease symptoms and/or regulatory dysfunction. Analysis of centrally acting monoamine concentrations in the endogenous state reveals a significant difference in day-to-day assays performed on the same subject with and without monoamine-related disease symptoms and/or regulatory dysfunction. The day-to-day difference renders baseline testing in the endogenous state non-reproducible in the same subject.

Conclusion: It is asserted that the monoamine hypothesis, which claims that low synaptic levels of monoamines are a primary etiology of disease, is not a valid primary reference point for understanding chronic electrical dysfunction related to the centrally acting monoamines. Furthermore, the "bundle damage theory" is a more accurate primary model for understanding chronic dysfunction. The "bundle damage theory" advocates that synaptic monoamine levels are normal but not adequate in states associated with chronic electrical dysfunction and that levels need to be increased to compensate for the chronic postsynaptic electrical dysfunction due to existing damage. The monoamine hypothesis, in failing to accurately explain the etiology of chronic neuronal electrical flow dysfunction in the endogenous state, is reduced to no more than a historical footnote.

Keywords: monoamine hypothesis, monoamine theory, serotonin, dopamine, neuronal dysfunction, bundle damage theory

\section{Introduction}

This paper is the continuation of a series of original research papers published by the authors on the topic of nutritionally driven organic cation transporter (OCT) functional status determination (herein referred to as OCT assay[s]). This paper correlates original research previously published by the authors on the topic of transporter-driven centrally acting monoamine observations with the monoamine hypothesis. ${ }^{1-12}$ 
The centrally acting monoamines serotonin, dopamine, norepinephrine and epinephrine (herein referred to as "monoamine[s]") exist in one of two states. The "endogenous state" is present when no supplemental amino acids are being administered, and the "competitive inhibition state" is found when significant amounts of serotonin and/or dopamine amino acid precursors are simultaneously administered. ${ }^{1-7}$

Previous literature described the competitive inhibition state as "functionally meaningless." The basis for this assertion was the inability to alter monoamine levels with amino acid precursors and then objectively quantify the changes. ${ }^{7}$ With the perfection of the novel OCT assay analysis by the authors, the competitive inhibition state is no longer functionally meaningless. ${ }^{1-12}$

Since the early 1960s, the monoamine hypothesis has been a reference point for understanding the etiology of the electrical defects associated with monoamine-related disease and the mechanism of action of reuptake inhibitors. The monoamine hypothesis posits that depression is caused by decreased monoamine function in the brain. The hypothesis originated from early empirical clinical observations and has been generally recognized to mean that low concentrations of synaptic monoamines are a primary factor in the etiology of depression, other monoamine-related disease states, and regulatory dysfunction. ${ }^{13}$

The bundle damage theory was first published in 2009. It advocates that although synaptic levels of monoamines are normal in chronic monoamine-related disease states, these levels are inadequate in compensating for postsynaptic damage to structures conducting electricity. ${ }^{8}$

In this manuscript, the new conclusions about monoamine hypothesis and the bundle damage theory are compared with the original research of the authors. When inadequate levels of monoamines exist, the only way to increase the total number of monoamine molecules in the brain is through administration of their amino acid precursors. This is because monoamines do not cross the blood-brain barrier. The amino acid precursors can cross the barrier, and are synthesized into new monoamines. Whether the synaptic levels are lower than normal or normal at the start of management, nutritional status is a primary consideration in addressing problems associated with inadequate monoamines. . $^{4,6,10}$

There are two primary types of nutritional deficiencies. The monoamine hypothesis advocates that an absolute nutritional deficiency (AND) is the core issue of monoaminerelated electrical dysfunction, whereas the bundle damage theory advocates a relative nutritional deficiency (RND). ${ }^{8}$
An AND occurs when not enough nutrients are included in the diet, leading to nutritional concentrations that are not adequate for establishing normal synaptic monoamine levels (the monoamine hypothesis). A relative nutritional deficiency occurs when synaptic levels are normal in the endogenous state but not high enough to compensate for damage to the postsynaptic neuronal structures that conduct electricity (the bundle damage theory).

The organic cation transporters (OCT) are primary determinants of intracellular and extracellular (including synaptic) monoamine concentrations. ${ }^{13}$ Previously published literature by the authors provides proof that in the endogenous state transporter-dependent monoamine concentrations are indistinguishable in subjects with and without monoamine-related disease and/or regulatory dysfunction. These findings are an integral part of the challenge to the validity of the monoamine hypothesis. ${ }^{4,6,10}$

\section{Methods and materials}

Original research results by the authors ${ }^{1-12}$ outlined a novel methodology for nutritionally driven OCT assay analysis that defines the phase of monoamine transport, status of transporter entrance gates, transporter lumen saturation status, and transporter balance status between the monoamines and their amino acid precursors. These are all critical to determining whether the relative concentrations of the centrally acting monoamines are being effectively transported. ${ }^{1-12}$

\section{Nutritionally driven OCT functional status determination}

Under normal conditions, serotonin and dopamine filtered at the glomerulus are metabolized by the kidneys, which prevent significant amounts of these peripheral monoamines from being found in the final urine. Urinary serotonin and dopamine, in subjects not suffering from a monoaminesecreting tumor, represent monoamines newly synthesized in the proximal convoluted renal tubule cells of the kidneys. These monoamines have never been in the central or peripheral systems. Once synthesized, their fate is dependent upon the interaction of the basolateral monoamine transporters (OCT2) and the apical monoamine transporters (OCTN2). The OCT2 transports serotonin and dopamine to the interstitium. These monoamines then end up in the peripheral system via the renal vein. The OCTN2 of the apical membrane transports the serotonin and dopamine not transported by the OCT2 to the proximal nephrons of the kidneys, before sending them to the urine as waste. Proper OCT assay requires that initially the serotonin and dopamine systems 
are placed in the competitive inhibition state simultaneously, while administering adequate amounts of serotonin and dopamine amino acid precursors. The assay results are then compared in order to determine the change in urinary serotonin and dopamine concentrations associated with changes in amino acid precursor dosing values. , $3,5,6,11^{-1}$

A urinary serotonin or dopamine value less than $80 \mu \mathrm{g}$ or $475 \mu \mathrm{g}$ of monoamine per gram of creatinine, respectively, is defined as a phase 2 response. A urinary serotonin or dopamine value greater than 80 or $475 \mu \mathrm{g}$ of monoamine per gram of creatinine, respectively, is interpreted as being in phase 1 or phase 3. Differentiation of phase 1 from phase 3 is as follows. If a direct relationship is found between amino acid dosing and urinary assay response, it is referred to as a phase 3 response. An inverse relationship is referred to as a phase 1 response. The phase 3 optimal range for urinary serotonin is defined as $80-240 \mu \mathrm{g}$ of serotonin per gram of creatinine. The phase 3 optimal range for urinary dopamine is defined as $475-1,100 \mu \mathrm{g}$ of dopamine per $\mathrm{g}$ of creatinine. , $^{2,3,5,6,11}$

Processing, management, and assay of the urine samples are as follows: urine samples are collected about 5-6 hours prior to bedtime, with 4:00 pm being the most frequent collection time point. The samples are stabilized in $6 \mathrm{~N} \mathrm{HCl}$ to preserve the dopamine and serotonin. The urine samples are collected after a minimum of 1 week, during which time the patient has been taking a specific daily dosing of amino acid precursors of serotonin and dopamine where no doses are missed. Samples are shipped to DBS Laboratories (Duluth, MN). Urinary dopamine and serotonin are assayed utilizing commercially available radioimmunoassay kits (3 CAT RIA IB88501 and IB89527, both from Immuno Biological Laboratories Inc, Minneapolis, MN). The DBS laboratory is accredited as a high-complexity laboratory by Clinical Laboratory Improvement Amendments (CLIA) to perform these assays. OCT assay interpretation is performed by one of the authors (Marty Hinz, MD, NeuroResearch Clinics, Inc).

\section{Results}

The authors previously published "matched pairs $t$-test" results for the transporter-dependent, centrally acting monoamine concentrations in the endogenous state from the same subject on different days. This current paper is a continuation of this discussion based on original research that expands on the scope and implications of these scientific findings within the context of the monoamine hypothesis., ${ }^{4,6,10}$

In this previously published original research, spot baseline urinary assays for each monoamine were obtained for the first test on day one and for the second test on a different day. Both occurred at the same time of the day for each subject. The two tests from each subject were then paired, and a statistically significant grouping of matched pairs was subjected to the "matched pairs $t$-test." The results are a critical component in forming the foundation of the conclusions in this paper. ${ }^{4,6,10}$

These original research studies reported that spot baseline urinary serotonin, dopamine, norepinephrine, and epinephrine concentrations in the endogenous state differ in a statistically significant manner from day to day in the same subject. This supports the conclusion that under normal conditions baseline urinary monoamine testing is not uniform or reproducible from day to day in the same subject. The functional status of these organic cation transporters determines intracellular and extracellular (including synaptic) concentrations of these monoamines. Furthermore, it was concluded that it is virtually impossible to distinguish, via laboratory assay interpretation, individuals with or without disease or regulatory dysfunctions, even those dysfunctions that were traditionally assumed to be associated with low levels of synaptic, centrally acting monoamine levels., ${ }^{4,6,10}$

\section{Discussion}

The monoamine hypothesis holds that low concentrations of synaptic monoamines are the primary etiology of monoamine-related chronic electrical dysfunction. ${ }^{13}$ The corollary to this premise is that returning synaptic monoamine levels to normal will resolve electrical dysfunction. In correlating the perspective of the monoamine hypothesis with peer-reviewed literature published by the authors since 2009, the following considerations and conclusions exist.

\section{Differentiation of those with and without disease}

There is no objective proof demonstrating that low in situ levels of centrally acting monoamine concentrations in the synapse are the primary etiology under normal conditions. ${ }^{14}$ There is no objective method that identifies individuals with low concentrations of transporter-dependent monoamine concentrations in the endogenous state. ${ }^{13}$ Transporter-associated concentration trends in groups of subjects have been identified, but the day-to-day variability of monoamine concentrations in each individual comprising the group reveals that it is not possible to identify individuals with electrical dysfunction on laboratory testing and/or transporter analysis who are suffering from low levels of monoamines relative to the normal reference range. ${ }^{4,6,11}$ Diets devoid of 
critical amino acids will induce an AND with associated disease symptoms, but diets such as this are not the normal endogenous state of humans who develop monoamine or regulatory dysfunction-related symptoms. ${ }^{15,16}$

Synaptic monoamine concentrations are primarily dependent on the functional status of the nutritionally driven organic cation transporters. The monoamines and their amino acid precursors are "organic cations" that are transported by the three primary electrogenic organic cation transporter types, each of which has several subtypes: OCT1, OCT2, and OCT3. The OCT of the liver, brain, kidney, and bowels are identical and homologous. ${ }^{17}$ Of the three transporter types, the OCT2 has tissue expression primarily in the kidney and the brain.

OCT assay analysis has led to the ability to define the phases of monoamine transport, transporter saturation status, the status of monoamine and precursor transporter balance, the amount of waste (unneeded) monoamines the transporters are excreting, and the status of transporter entrance gates. After doing this OCT assay analysis, we can define the individualized amino acid dosing values needed for optimal flow of electricity through damaged postsynaptic bundles as evidenced by clinical outcomes. ${ }^{12,13}$

There is no objective documentation that identifies individuals with low concentrations of transporter-dependent monoamine concentrations in the endogenous state. Transporter-associated concentration trends in groups of subjects have been identified, but the day-to-day variability of monoamine concentrations in each individual comprising the group reveals that it is not possible to identify individuals with electrical dysfunction on laboratory testing and/or transporter analysis who are suffering from low levels of monoamines relative to the normal reference range. ${ }^{4,6,10}$

In the endogenous state, under the monoamine hypothesis, low synaptic concentrations of monoamines are a primary cause of electrical dysfunction. ${ }^{14}$ If this were true, the significant fluctuations in transporter-dependent monoamine concentrations from day to day in the individual should lead to clinical states where the findings would wax and wane in a manner consistent with day-to-day observed fluctuations in transporter-driven monoamine concentrations as documented in same subject studies (matched pairs $t$-test). This is not the case. The etiology of chronic problems is not low concentrations of monoamines that need to be returned to normal as predicted by the monoamine hypothesis; it is concentrations that are normal but not high enough to compensate for postsynaptic neuronal damage. Addressing this electrical defect properly requires the system to be placed into the competitive inhibition state in order to be able to increase monoamine levels to above normal to reach the threshold level needed to establish the adequate electrical flow required. Analysis of transporter-driven monoamine needs reveals that postsynaptic electrical conduction damage in patients with chronic disease is so high that the day-to-day monoamine fluctuations of the endogenous state are below the threshold needed to attain symptom relief. Therefore, chronic symptoms do not wax and wane as might be predicted by the laboratory results obtained in the endogenous state..$^{5,8}$ Previous writings of the authors demonstrated relative nutritional deficiencies in Parkinson's disease,${ }^{5}$ chronic depression, ${ }^{9,12}$ Crohn's disease, ${ }^{2}$ and attention deficit hyperactivity disorder without any findings that would support an AND. ${ }^{3}$ Restoration of regulatory function in these RND conditions is only possible when transporterdependent monoamine concentrations are elevated above normal and properly balanced in the competitive inhibition state (see Figure 1). ${ }^{1-12}$

Many disease states have been recognized as having a common etiology of postsynaptic bundle damage associated with insult. ${ }^{1-12}$ Different areas of damage to the nerve bundles result in different disease entities. These entities all share a common pathology of inadequate levels of the monoamine-driven electrical activity that is required to power the functions of the body. This results in the relative nutritional deficiency that requires monoamine levels higher than normally found in the synapse to overcome the damaged areas of the nerve bundles. Parkinson's disease demonstrates this deficiency and the ability of targeted amino acid precursor supplementation to restore function. ${ }^{5}$

\section{Parkinson's disease as a prototype}

Parkinson's disease is a prototype disease that illustrates the mechanism of action of postsynaptic neuron damage and its compensation. Chronic damage to the postsynaptic dopamine fibers of the substantia nigra induce an RND that is not just dopamine related but is related to all of the centrally acting monoamines. This RND causes Parkinson's disease symptoms by compromising the flow of electricity regulating fine motor control. The monoamine levels of Parkinson's patients prior to treatment are found to be in the normal range. Proper management of Parkinson's disease requires an increase in the synaptic levels of dopamine with a higher than normal administration of L-dopa. Increasing the synaptic neurotransmitter with L-dopa is analogous to turning up the voltage. It causes more electricity to flow through the remaining viable postsynaptic, electricity-conducting neuronal structures. 
When enough electricity is once again flowing, control of symptoms is effected. ${ }^{5}$

\section{Dietary management}

The monoamines do not cross the blood-brain barrier. The only way to increase the total number of monoamine molecules in the brain - to a level that is higher than is possible with dietary modification - is with supplemental nutritional support through administration of properly balanced amino acid precursors and cofactors. These cross the blood-brain barrier and are synthesized into new monoamines. ${ }^{1-12}$

The immediate amino acid precursors of serotonin and dopamine, 5-HTP and L-dopa, respectively, freely cross the blood-brain barrier to synthesize into their respective monoamines without biochemical feedback inhibition (Figure 2). At equilibrium the amino acid precursors have a similar effect on all identical and homologous OCTs and subtypes throughout the body. ${ }^{12}$

When synapse-related electrical compromise is present, the monoamine hypothesis advocates that an AND exists, ie, low synaptic monoamine levels are present and returning these levels to normal will restore adequate electrical flow. This would predict that an optimized normal diet, with no supplemental nutrients, will restore the low levels of synaptic monoamines back to normal, leading to relief of the electrical dysfunction that is causing the disease or regulatory dysfunction. This does not happen. Literature has not described dietary modification as a valid and/or effective approach in management of monoamine-related synaptic electrical dysfunction under normal conditions.

Under the bundle damage theory, as discussed in the next section, when neuronal electrical compromise (due to postsynaptic damage) is significant, a relative nutritional deficiency is concomitantly present. Proper compensation requires that the system be placed in the competitive inhibition state where synaptic monoamine levels are higher than normal; this cannot be achieved with dietary modification alone. Administration of properly balanced supplemental amino acid precursors

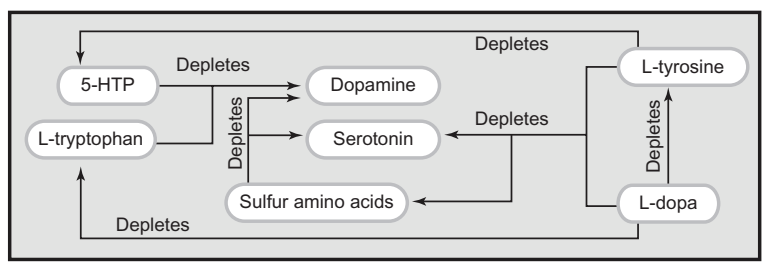

Figure I If dopamine precursors (L-tyrosine and/or L-dopa) are not in proper balance with serotonin precursors (5-HTP and/or L-tryptophan), depletion of serotonin or dopamine will occur. All components of the system need to be in proper balance. under the guidance of OCT assay analysis is needed. This ensures proper amino acid and monoamine transport balance and compensates for the electrical defect.

The balance between serotonin precursors, dopamine precursors, and sulfur amino acids is critical, as profound interactions exist between these substances. When administration of these substances is not in proper balance, an additional amino acid-induced RND develops (see Figure 1). ${ }^{1-12}$

There are many things that can be gleaned out of Figure 1, such as administering only 5-HTP facilitates depletion of dopamine. Giving only L-dopa facilitates depletion of serotonin, sulfur amino acids, L-tyrosine, and L-tryptophan.

The administration of properly balanced 5-HTP with L-dopa establishes transporter-dependent synaptic monoamine concentrations at levels higher than normal. These levels compensate for the relative nutritional deficiency and resultant electrical deficit. ${ }^{18}$ In contrast, the monoamine hypothesis has never demonstrated that under normal conditions returning synaptic monoamine levels to normal is effective.

\section{The bundle damage theory}

Under the bundle damage theory, relative nutritional deficiency is the cause of chronic electrical dysfunction observed with centrally acting monoamine-related problems. This is supported by the fact that in the endogenous state all subjects with and without disease have similar and indistinguishable monoamine levels. The primary source of the chronic electrical dysfunction under the bundle damage theory is damage to the postsynaptic structural components involved with electrical conduction. In this state, the levels of synaptic monoamines are normal and an RND exists. ${ }^{8}$

A list of almost 1200 known neurotoxins found in the environment serves as a backdrop for this discussion. ${ }^{19}$ Neurotoxins, trauma, biologics, and/or genetic predisposition contribute to postsynaptic structural damage which compromises electrical flow when synaptic monoamine levels are normal. This damage tends to be cumulative. The flow of electricity between the pre- and postsynaptic neurons is mediated by synaptic levels of centrally acting monoamines. This causes electrically dependent functions to be improperly regulated. ${ }^{5,8}$

Individual dendrite structures of postsynaptic neurons do not facilitate electrical flow as a single entity. Multiple postsynaptic structures, functioning as bundles, regulate function. The bundle damage theory states that a significant factor in the development of monoamine-related electrical dysfunction disease or regulatory dysfunction 


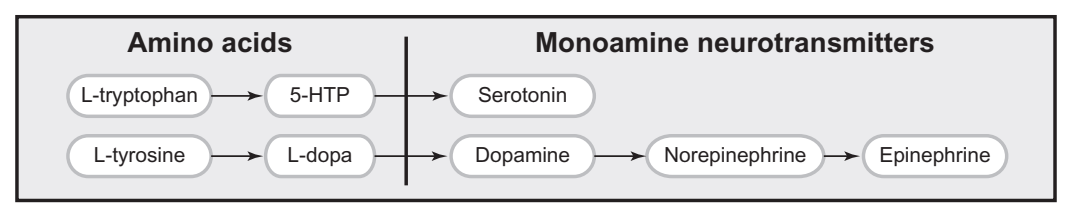

Figure $\mathbf{2}$ The centrally acting monoamines with their amino acid precursors.

occurs when the electrical flow through the postsynaptic neuron bundles regulating function is compromised by damage. In order to optimally restore neuron bundle regulatory function, synaptic neurotransmitter levels involved with transference of electrical flow across the synapse into the remaining viable postsynaptic neuron structures must be increased to levels higher than are normally found in the system. This in turn results in restoration of adequate electrical flow, relief of symptoms, and/or resolution of regulatory dysfunction. ${ }^{8}$

Support for the bundle damage theory is that restoration of normal neuronal electrical flow can be accomplished by increasing monoamine concentrations into the competitive inhibition state, where organic cation transporter-driven and synaptic monoamine concentrations are higher than those found in the endogenous state. The situation is managed as a relative nutritional deficiency. Instead of ascribing the symptom etiology to low concentrations of transporter-dependent synaptic monoamines in chronic states, it is more accurate to attribute the cause to synaptic monoamine concentrations being chronically inadequate to compensate for electrical dysfunction induced by postsynaptic structural damage. When chronic monoamine-related deficiency states exist, this terminology more appropriately explains the need to increase synaptic monoamine concentrations into the competitive inhibition state. ${ }^{4,6,10}$

The World Health Organization's observation, consistent with the bundle damage theory, is that higher toxicant exposure (in developed countries) contributes to the higher rate of depression and other monoamine-related disease. ${ }^{8}$

Relative nutritional deficiency, secondary to postsynaptic structural damage, may be the only issue in which proper management allows for removing the RND from the clinical picture. This is to ensure that any other possible concomitant disease and regulatory dysfunction etiologies or mechanisms of action may be focused on more clearly.

\section{Reuptake inhibitors}

The mechanism of action of reuptake inhibitors is unknown, but it is theorized that blocking of transporter reuptake leads to increased concentrations of synaptic monoamines and restoration of electrical flow. Reuptake inhibitor efficacy in the treatment of depression is low. Double-blind, placebocontrolled studies consistently reveal reuptake inhibitor depression efficacy of $7 \%$ to $13 \%$ greater than placebo. From another perspective, this means $87 \%$ to $93 \%$ of patients treated with reuptake inhibitors for depression can expect to achieve results no greater than placebo. The authors previously reported the novel findings that the effects of reuptake inhibitors on transporter-driven monoamine concentrations revealed serotonin concentrations changed $<50 \mu \mathrm{g} / \mathrm{gr}$ creatinine. These very small OCTdriven changes in monoamine concentrations are consistent with the low efficacy of the reuptake inhibitors. Group analysis shows no statistical significance. ${ }^{9,18}$

If simply establishing synaptic monoamine concentrations in the normal range under an absolute nutritional deficiency approach as predicted by the monoamine hypothesis were all that is required, it would be expected that reuptake inhibitor efficacy would be higher than reported. This is not the case. In previously published manuscripts by the authors, subjects with depression were managed under the relative nutritional deficiency approach using monoamine precursor nutritional support with 5-HTP and L-dopa which elevated the mean serotonin and dopamine concentrations higher than normal into the desired competitive inhibition phase 3 range, leading to restoration of electrical flow. This procedure produced magnitudes of increased levels of the transporter-driven serotonin and dopamine concentrations, far beyond the increases observed with reuptake inhibitors alone. ${ }^{1-12}$

The required serotonin and dopamine precursor dosing values are independent of each other in the competitive inhibition state. Optimal daily ranges exist when all monoaminerelated diseases are examined in the competitive inhibition state. Some variances of the high end of the range may occur when the individual diseases are examined. The 5-HTP daily effective therapeutic range is $>0 \mathrm{mg}$ to $2400 \mathrm{mg}$. The L-dopa daily effective therapeutic range (in subjects not suffering from Parkinson's disease or Restless Leg Syndrome) is $>0 \mathrm{mg}$ to $2100 \mathrm{mg}$. The tyrosine daily effective therapeutic range is $>0 \mathrm{mg}$ to $14,000 \mathrm{mg} \cdot{ }^{1-12}$ 
Table I A comparison of the monoamine hypothesis and the bundle damage theory

\begin{tabular}{|c|c|c|}
\hline & Monoamine hypothesis & Bundle damage theory \\
\hline $\begin{array}{l}\text { Synaptic monoamine levels when } \\
\text { electrical dysfunction exits }\end{array}$ & Low & Normal \\
\hline Neuronal system status & Normal & $\begin{array}{l}\text { Postsynaptic structural damage leading to } \\
\text { compromised electrical flow }\end{array}$ \\
\hline $\begin{array}{l}\text { Monoamine levels required } \\
\text { to restore electrical flow }\end{array}$ & Normal (endogenous state) & Higher than normal (competitive inhibition state) \\
\hline Etiology & Nutritional deficiency & $\begin{array}{l}\text { Recurrent damage due to neurotoxins, trauma, } \\
\text { biologics and/or genetic predisposition }\end{array}$ \\
\hline $\begin{array}{l}\text { Conclusion on the } \\
\text { basis of the etiology }\end{array}$ & $\begin{array}{l}\text { Absolute nutritional deficiency, dietary modification } \\
\text { (no supplements) will correct the problem }\end{array}$ & $\begin{array}{l}\text { Relative nutritional deficiency, properly } \\
\text { balanced supplementation needed to establish } \\
\text { monoamine levels higher than normal }\end{array}$ \\
\hline Laboratory observations & $\begin{array}{l}\text { From a laboratory standpoint, in the endogenous state, } \\
\text { unable to distinguish those with and without disease } \\
\text { contrary to predictions of the monoamine hypothesis }\end{array}$ & $\begin{array}{l}\text { OCT assay determination in the competitive } \\
\text { inhibition state allows for predictable outcomes } \\
\text { to nutritionally driven monoamine changes }\end{array}$ \\
\hline Undermining the concept & $\begin{array}{l}\text { Literature has never described dietary modification } \\
\text { that simply returns synaptic monoamine levels } \\
\text { to normal as a valid approach in management of } \\
\text { monoamine-related electrical dysfunction }\end{array}$ & None \\
\hline Support for the concept & $\begin{array}{l}\text { Empirical observations that increasing synaptic } \\
\text { monoamine levels leads to clinical improvement } \\
\text { without proof that simply returning monoamine } \\
\text { levels to normal is what is happening }\end{array}$ & $\begin{array}{l}\text { Published literature on difficult to treat cases of } \\
\text { Parkinson's disease, chronic depression, Crohn's } \\
\text { disease, attention deficit hyperactivity disorder, } \\
\text { where synaptic levels are initially normal then } \\
\text { intentionally increased to higher than normal } \\
\text { to compensate for chronic electrical damage }\end{array}$ \\
\hline
\end{tabular}

Abbreviation: OCT, organic cation transporter.

Table 1 juxtaposes the monoamine hypothesis against the bundle damage theory.

\section{Conclusion}

The authors of this manuscript have published more than a dozen peer-reviewed papers on the topic of centrally acting monoamines and administration of their precursors. This paper correlates previous original research findings of the authors with the monoamine hypothesis and is a continuation of the scientific discussion. ${ }^{1-12}$ While there has been previous literature that has discredited the monoamine hypothesis, this paper sheds further light on the topic.

The monoamine hypothesis is based on the assumption that synaptic concentrations of monoamines are lower than normal in monoamine-related, central neuronal electrical dysfunction states. This supports the assertion that addressing the problem under an absolute nutritional deficiency strategy by returning synaptic monoamine concentrations to normal would be effective. The contents of this paper prove that this does not happen. ${ }^{13}$

The bundle damage theory states that monoamine concentrations are normal but not adequate, due to an RND in subjects with and without chronic disease. In order to restore adequate electrical flow and compensate for postsynaptic damage, organic cation transporter-driven synaptic monoamine levels must be increased to a level greater than those concentrations found in the endogenous state, under a monoamine amino acid RND approach outlined in previous peer-reviewed original research publications. ${ }^{8}$

The key difference between the monoamine hypothesis and the bundle damage theory is the perception that electrical dysfunction is caused by low synaptic concentrations of monoamines versus normal synaptic concentrations of monoamines that are not high enough to compensate for postsynaptic structural damage.

Analysis of monoamine concentrations in subjects in the endogenous state with and without the presence of monoamine-related electrical dysfunction reveals that it is impossible to differentiate these subjects based on laboratory testing. ${ }^{4,6,10}$

Reuptake inhibitors have low efficacy in the treatment of monoamine-related disease. Their focus is treatment of the disease without addressing the proper balance of monoamines and precursors required under the relative nutritional deficiency approach. This is consistent with findings that reuptake inhibitors cause no statistically significant changes in transporter-dependent monoamine concentrations. ${ }^{12}$

In chronic disease states the leading cause of electrical dysfunction is monoamine-related RND, secondary to damage to postsynaptic neuronal structures caused by 
neurotoxins, trauma, biologics, and/or genetic predisposition. The only way to compensate for damaged electrical flow is to properly balance serotonin and dopamine in the competitive inhibition state through administration of amino acid precursors under the guidance of OCT assay determination. . $^{2,3,5,7,8}$

Postsynaptic electrical dysfunction may not be the only etiology of monoamine-related dysfunction. Proper administration of serotonin and dopamine amino acid precursors, under the guidance of OCT assay determination, removes concerns of RND from the clinical picture, facilitating the ability to clearly focus on other possible etiologies as needed.

The monoamine hypothesis is simply not a valid concept. It is the goal of this manuscript to stimulate interest and dialogue regarding the etiology of synaptic monoamineassociated electrical dysfunction.

\section{Disclosure}

$\mathrm{MH}$ discloses ownership of NeuroResearch Clinics, Inc. TU discloses lab directorship of DBS Labs, Duluth, MN. AS reports no conflict of interest related to this paper.

\section{References}

1. Hinz M, Stein A, Uncini T. The dual-gate lumen model of renal monoamine transport. Neuropsychiatr Dis Treat. 2010;6:387-392.

2. Stein A, Hinz M, Uncini T. Amino acid responsive Crohn's disease, a case study. Clin Exp Gastroenterol. 2010;3:171-177.

3. Hinz M, Stein A, Uncini T. Treatment of attention deficit hyperactivity disorder with monoamine amino acid precursors and organic cation transporter assay interpretation. Neuropsychiatr Dis Treat. 2011;7:31-38.

4. Hinz M, Stein A, Uncini T. Urinary neurotransmitter testing: considerations of spot baseline norepinephrine and epinephrine. Open Access Journal of Urology. 2011;3:19-24.
5. Hinz M, Stein A, Uncini T. Amino acid management of Parkinson disease: a case study. Int J Gen Med. 2011;4:1-10.

6. Hinz M, Stein A, Uncini T. Validity of urinary monoamine assay sales under the "spot baseline urinary neurotransmitter testing marketing model". Int J Nephrol Renovasc Dis. 2011;4:101-113.

7. Hinz M, Stein A, Uncini T. APRESS: apical regulatory super system, serotonin, and dopamine interaction. Neuropsychiatr Dis Treat. 2011;7:1-7.

8. Hinz M. Depression. In: Kohlstadt I, editor. Food and Nutrients in Disease Management Boca Raton: CRC Press; 2009:465-481.

9. Trachte G, Uncini T, Hinz M. Both stimulatory and inhibitory effects of dietary 5-hydroxytryptophan and tyrosine are found on urinary excretion of serotonin and dopamine in a large human population. Neuropsychiatr Dis Treat. 2009;5:227-235.

10. Hinz M, Stein A, Trachte G, Uncini T. Neurotransmitter testing of the urine; a comprehensive analysis. J Urol. 2010;2:177-183.

11. Hinz M, Stein A, Uncini T. A pilot study differentiating recurrent major depression from bipolar disorder cycling on the depressive pole. Neuropsychiatr Dis Treat. 2010;6:741-747.

12. Hinz M, Stein A, Uncini T. Monoamine depletion by reuptake inhibitors. Journal of International Drug, Healthcare and Patient Safety. 2011;3: 69-77.

13. Krishnan V, Nestler E. The molecular neurobiology of depression. Nature. 2008;455:902-984.

14. Soares-da-silva P, Pinto-do-O PC. Antagonistic actions of renal dopamine and 5-hydroxytryptamine: effects of amine precursors on the cell inward transfer and decarboxylation. Br J Pharmacol. 1996;117:1187-1192.

15. Young S, Smith S, Pihl R. Tryptophan depletion causes a rapid lowering of mood in normal males. Psychopharmacology.1987;2:173-177.

16. Smith K, Fairburn C, Cowen D. Relapse of depression after rapid depletion of tryptophan. Lancet. 1997;349(9056):915-919.

17. Koepsell H. Organic cation transporters in the intestine, kidney, liver, and brain. Annu Rev Physiol. 1998;60:243-266.

18. Scorecard.org. Neurotoxicants. Washington, DC: Green Media Toolshed. Available from: http://scorecard.goodguide.com/healtheffects/chemicals-2.tcl?short_hazard_name=neuro\&all_p=t. Accessed December 16, 2011.

19. Koepsell H, Schmitt B, Gorboulev V. Organic cation transporters. Rev Physiol Biochem Pharmacol. 2003;150:36-90.
International Journal of General Medicine

\section{Publish your work in this journal}

The International Journal of General Medicine is an international, peer-reviewed open-access journal that focuses on general and internal medicine, pathogenesis, epidemiology, diagnosis, monitoring and treatment protocols. The journal is characterized by the rapid reporting of reviews, original research and clinical studies across all disease areas.

\section{Dovepress}

A key focus is the elucidation of disease processes and management protocols resulting in improved outcomes for the patient.The manuscript management system is completely online and includes a very quick and fair peer-review system. Visit http://www.dovepress.com/ testimonials.php to read real quotes from published authors. 